\title{
Scaling and aging in the homogeneous cooling state of a granular fluid of hard particles
}

\author{
J.J. Brey, A. Prados, M.I. García de Soria, and P. Maynar \\ Física Teórica, Universidad de Sevilla, \\ Apartado de Correos 1065, E-41080, Sevilla, Spain
}

(Dated: November 4, 2018)

\begin{abstract}
The presence of the aging phenomenon in the homogeneous cooling state (HCS) of a granular fluid composed of inelastic hard spheres or disks is investigated. As a consequence of the scaling property of the $N$-particle distribution function, it is obtained that the decay of the normalized twotime correlation functions slows down as the time elapsed since the beginning of the measurement increases. This result is confirmed by molecular dynamics simulations for the particular case of the total energy of the system. The agreement is also quantitative in the low density limit, for which an explicit analytical form of the time correlation function has been derived. Moreover, the reported results provide support for the existence of the HCS as a solution of the N-particle Liouville equation.
\end{abstract}

PACS numbers: 45.70.-n,51.10.+y,05.20.Dd 


\section{INTRODUCTION}

In many far from equilibrium states, it has been observed that the relaxation (or response) rate decreases as the "age" of the process increases. Here "age" refers to the time elapsed since the beginning of the considered experiment. Then, it is said that the system ages, or that it exhibits an aging phenomenon. A revision of the concept of aging in spin glasses and in other systems can be found in ref. [1] .

One of the typical experiments for the analysis of aging in a given physical system is the study of the two-time correlation functions of some of its properties, since they are related with the response of the system to a given perturbation. Let $C_{A B}\left(t_{w}, t\right)$ denote the two-time correlation function of the magnitudes $A$ and $B$ of the system, the former being measured at time $t_{w}$ and the latter at time $t \geq t_{w}$. In a system at equilibrium, $C_{A B}\left(t_{w}, t\right)$ only depends on the the time difference $\tau=t-t_{w}$, due to the time translational invariance. On the other hand, in systems presenting aging, it depends on both $\tau$ and $t_{w}$. It is important to stress that the aging phenomenon is more than just the loss of the time translational invariance; it consists in the relaxation of $C_{A B}\left(t_{w}, t_{w}+\tau\right)$ slowing down as the "waiting time" $t_{w}$ increases.

The simplest aging phenomenon occurs when the two-time correlation function depends only on the time ratio $\tau / t_{w}$, and it is sometimes called "full aging". This behavior is exhibited by some simple models, such as mean field model of spin glasses [2, 3] and the one-dimensional Ising model at zero temperature [4, 5]. More complicated dependencies like $\ln t / \ln t_{w}$ have also been found [6] and, with more generality, behaviors of the form $h(t) / h\left(t_{w}\right)$, with different functions $h[1,7]$.

Granular media are inherently non-equilibrium systems, due to the dissipative character of the interactions between grains. There is a continuous loss of kinetic energy and the system tends to a rest, unless energy is being continuously injected into the system, for instance through a vibrating wall. A kind of typical experiments carried out in dense granular systems are those designed to investigate compaction [8]. Usually, the system is submitted to a series of separated pulses or taps of a given short duration. After each tap, the system is allowed to relax freely until it reaches a metastable configuration with all the particles at rest. In this state, there are many permanent contacts between particles. Next, the system is tapped again and the process is repeated many times. Properties of interest like the volume or the energy are measured at each rest configuration. In this way, the evolution of these 
properties as a function of time, measured in number of taps, is obtained. For large number of taps, the system tends to a steady state with a density that is a monotonically decreasing function of the tapping intensity [8, 9]. The relaxation of the system towards the steady state is very slow and clearly non-exponential. Furthermore, when the intensity of tapping is changed cyclically, hysteresis phenomena in the density are observed [9]. These behaviors are similar to those found in structural glasses when submitted to cyclical variations of their temperature [10] and are a quite strong evidence of the presence of aging phenomena in compact granular media. Actually, aging has been observed both experimentally [11] and also identified in simple models of compaction [12, 13].

A completely different regime of granular systems are the so-called granular gases, in which there are not permanent contacts between particles, but they move freely and independently between collisions. The simplest possible state of a granular gas is the homogeneous cooling state (HCS), whose temperature decays monotonically in time. At a microscopic level, the HCS is assumed to be characterized by a phase space probability distribution in which all the time dependence occurs through the temperature. For the case of hard particles, this implies a scaling property and the possibility of identifying some features of the time dependence of many relevant properties of the system, without carrying out explicit calculations. Among these properties are ensemble averages as well as time-correlation functions [14, 15]. The above peculiarities render the HCS a good candidate to investigate in detail and by means of analytical methods the possible existence of aging and, in the case of a positive answer, its origin and properties. In spite of the above, it has not been until very recently that attention has been devoted to this particular aspect of the HCS [16]. The aim of this paper is twofold. Firstly, to investigate in detail the possible existence of the aging phenomenon in the HCS of a granular fluid of inelastic hard particles, deepening into its origin. This is done both analytically and by means of particle simulation methods. The theoretical analysis will be based on the existence of the HCS at the level of the $N$-particle distribution function and its scaling property, for arbitrary density and inelasticity. Then, the accuracy of the predictions following from the theory provides strong support for the existence of the HCS in the context of a many body theory. This is precisely the second aim of the paper.

The presentation here proceeds as follows. In Sec. III some general properties of the $N$-particle distribution function defining the assumed HCS of a system of inelastic hard 
spheres or disks are shortly reviewed. By means of an appropriate scaling of the dynamics of the system, general features of the time dependence of the time-correlation functions can be identified. This is discussed in Sec. III, and the results presented hold, in principle, for a general pair of dynamical variables and arbitrary density and inelasticity. It is shown that the system exhibits full aging as a direct consequence of the scaling property of the HCS. The particular case of the total energy of the system is considered in Sec. IV]. The time selfcorrelation function of this property in the HCS is known in detail quite accurately in the low density limit, then allowing to get very detailed information about its time behavior. Comparison of the theoretical predictions with molecular dynamics simulation results is also presented. A quite good agreement is observed. Finally, Sec. V contains some general comments and final remarks.

\section{THE HOMOGENEOUS COOLING STATE OF A GRANULAR FLUID}

Consider a system on $N$ inelastic hard spheres $(d=3)$ or disks $(d=2)$ of mass $m$ and diameter $\sigma$. The position and velocity of particle $r$ at time $t$ will be denoted by $\boldsymbol{q}_{r}(t)$ and $\boldsymbol{v}_{r}(t)$, respectively. The dynamics of the system consists of free streaming, i.e. straight line motion along the direction of the velocity until a pair of particles, $r$ and $s$, is at contact, at which time their velocities $\boldsymbol{v}_{r}, \boldsymbol{v}_{s}$ change instantaneously to $\boldsymbol{v}_{r}^{\prime}, \boldsymbol{v}_{s}^{\prime}$ according to

$$
\begin{aligned}
& \boldsymbol{v}_{r}^{\prime}=\boldsymbol{v}_{r}-\frac{1+\alpha}{2}\left(\widehat{\boldsymbol{\sigma}} \cdot \boldsymbol{v}_{r s}\right) \widehat{\boldsymbol{\sigma}} \\
& \boldsymbol{v}_{s}^{\prime}=\boldsymbol{v}_{s}+\frac{1+\alpha}{2}\left(\widehat{\boldsymbol{\sigma}} \cdot \boldsymbol{v}_{r s}\right) \widehat{\boldsymbol{\sigma}}
\end{aligned}
$$

where $\boldsymbol{v}_{r s} \equiv \boldsymbol{v}_{r}-\boldsymbol{v}_{s}$ is the relative velocity and $\widehat{\boldsymbol{\sigma}}$ is a unit vector along $\boldsymbol{q}_{r s} \equiv \boldsymbol{q}_{r}-\boldsymbol{q}_{s}$ at contact. Finally, $\alpha$ is the coefficient of normal restitution, defined in the range $0<\alpha \leq 1$ and that will be considered here as a velocity-independent constant. The sequence of free streaming and binary collisions determines a unique trajectory of the system.

A macroscopic state is specified, at the statistical mechanics level, in terms of a probability density $\rho(\Gamma, t)$, with $\Gamma$ denoting a point in the $2 N d$ dimensional phase space of the system, $\Gamma \equiv\left\{\boldsymbol{q}_{1}, \boldsymbol{v}_{1}, \ldots, \boldsymbol{q}_{N}, \boldsymbol{v}_{N}\right\}$. The macroscopic variables of interest are the average of microscopic observables $A(\Gamma)$ at a given time $t$, defined in the two equivalent forms

$$
\langle A(t)\rangle \equiv \int d \Gamma \rho(\Gamma) e^{t L} A(\Gamma)=\int d \Gamma A(\Gamma) e^{-t \bar{L}} \rho(\Gamma) .
$$


In the above expressions, $L$ is the generator of the dynamics for phase functions, while $\bar{L}$ is the generator of the dynamics for distribution functions. Their expressions are

$$
\begin{gathered}
L(\Gamma) \equiv \sum_{r=1}^{N} \boldsymbol{v}_{r} \cdot \frac{\partial}{\partial \boldsymbol{q}_{r}}+\frac{1}{2} \sum_{r=1}^{N} \sum_{s \neq r}^{N} T(r, s), \\
\bar{L}(\Gamma) \equiv \sum_{r=1}^{N} \boldsymbol{v}_{r} \cdot \frac{\partial}{\partial \boldsymbol{q}_{r}}-\frac{1}{2} \sum_{r=1}^{N} \sum_{s \neq r}^{N} \bar{T}(r, s),
\end{gathered}
$$

with the binary collisions operators $T(r, s)$ and $\bar{T}(r, s)$ defined by

$$
\begin{gathered}
T(r, s) \equiv \sigma^{d-1} \int d \widehat{\boldsymbol{\sigma}} \Theta\left(-\widehat{\boldsymbol{\sigma}} \cdot \boldsymbol{v}_{r s}\right)\left|\widehat{\boldsymbol{\sigma}} \cdot \boldsymbol{v}_{r s}\right| \delta\left(\boldsymbol{q}_{r s}-\boldsymbol{\sigma}\right)\left(b_{r s}-1\right) \\
\bar{T}(r, s) \equiv \sigma^{d-1} \int d \widehat{\boldsymbol{\sigma}} \Theta\left(\widehat{\boldsymbol{\sigma}} \cdot \boldsymbol{v}_{r s}\right)\left|\widehat{\boldsymbol{\sigma}} \cdot \boldsymbol{v}_{r s}\right|\left[\alpha^{-2} \delta\left(\boldsymbol{q}_{r s}-\boldsymbol{\sigma}\right) b_{r s}^{-1}-\delta\left(\boldsymbol{q}_{r s}+\boldsymbol{\sigma}\right)\right] .
\end{gathered}
$$

In these expressions, $d \widehat{\boldsymbol{\sigma}}$ is the solid angle element corresponding to $\widehat{\boldsymbol{\sigma}}, \boldsymbol{\sigma} \equiv \sigma \widehat{\boldsymbol{\sigma}}, \boldsymbol{q}_{r s} \equiv \boldsymbol{q}_{r}-\boldsymbol{q}_{s}$, and $\Theta(x)$ is the Heaviside step function. Moreover, $b_{r s}$ is the substitution operator that replaces the velocities $\boldsymbol{v}_{r}$ and $\boldsymbol{v}_{s}$ to its right by their "postcollisional" values accordingly with Eqs. (11) and (2). Thus for an arbitrary function $F$,

$$
b_{r s} F\left(\boldsymbol{v}_{r}, \boldsymbol{v}_{s}\right)=F\left(\boldsymbol{v}_{r}^{\prime}, \boldsymbol{v}_{s}^{\prime}\right)
$$

Finally, the operator $b_{r s}^{-1}$ is the inverse of $b_{r s}$, i.e. it changes the velocities $\boldsymbol{v}_{r}, \boldsymbol{v}_{s}$ by their "precollisional" values,

$$
\begin{gathered}
b_{r s}^{-1} F\left(\boldsymbol{v}_{r}, \boldsymbol{v}_{s}\right)=F\left(\boldsymbol{v}_{r}^{\prime \prime}, \boldsymbol{v}_{s}^{\prime \prime}\right), \\
\boldsymbol{v}_{r}^{\prime \prime}=\boldsymbol{v}_{r}-\frac{1+\alpha}{2 \alpha}\left(\widehat{\boldsymbol{\sigma}} \cdot \boldsymbol{v}_{r s}\right) \widehat{\boldsymbol{\sigma}}, \\
\boldsymbol{v}_{s}^{\prime \prime}=\boldsymbol{v}_{r}+\frac{1+\alpha}{2 \alpha}\left(\widehat{\boldsymbol{\sigma}} \cdot \boldsymbol{v}_{r s}\right) \widehat{\boldsymbol{\sigma}} .
\end{gathered}
$$

In summary, the dynamics of the probability distribution function in phase space is governed by the Liouville equation

$$
\left(\frac{\partial}{\partial t}+\bar{L}\right) \rho(\Gamma, t)=0 .
$$

Due to the energy dissipation in collisions, there is no stationary solution to the above Liouville equation, except in the elastic limit $\alpha=1$. A granular temperature $T$ is usually defined from the average of the energy density. For a homogeneous state of a system composed of hard particles, it is given by

$$
T(t)=\frac{2}{N d}\langle E(t)\rangle
$$


with $E$ being the total (kinetic) energy of the system. By using Eq. (3), it is found

$$
\frac{\partial T(t)}{\partial t}=-\zeta(t) T(t)
$$

where the "cooling rate" $\zeta(t)$ is identified as

$$
\zeta(t)=-\frac{2}{T(t) N d}\langle L E(t)\rangle \geq 0 .
$$

Of course, there is a large class of time-dependent homogeneous states, depending on the initial preparation. Here, it will be assumed that, after a few collisions per particle, there is a relaxation of the velocity distribution towards a "universal" form, characterized because its entire time dependence occurs through the cooling temperature. This special state is called the homogeneous cooling state (HCS) and, at the macroscopic level, it is defined by a uniform number density $n_{h}$, a uniform but time-dependent temperature $T_{h}(t)$, and a vanishing flow velocity. Because of the absence of any additional microscopic energy scale for hard particles, its distribution function has the form

$$
\rho_{h}(\Gamma, t)=\left[\ell v_{0}(t)\right]^{-N d} \rho_{h}^{*}\left(\left\{\frac{\boldsymbol{q}_{r s}}{\ell}, \frac{\boldsymbol{v}_{r}}{v_{0}(t)} ; r, s=1, \ldots, N\right\}\right),
$$

where $v_{0}(t) \equiv\left(2 T_{h} / m\right)^{1 / 2}$ is a thermal velocity and $\ell \equiv\left(n_{h} \sigma^{d-1}\right)^{-1}$ a characteristic length proportional to the mean free path. The above special form of the $N$-particle distribution function allows to determine the temperature (and time) dependence of many average properties without explicit calculations. This fact will be actually exploited in the following.

The existence of the HCS solution to the Liouville equation has already been assumed several times in the literature [17, 18]. Although there is no direct proof of it, nor a constructive solution of the Liouville equation for this state has been developed, molecular dynamics (MD) simulations have shown that some of its implications, e.g. the scaling law for the temperature mentioned below, are observed in detail. Additional support has been provided by means of a time scale change that transforms the assumed HCS distribution into a time-independent distribution [19, 20]. MD simulations seem to confirm the existence of the steady state, that is reached after a few collisions per particle. A more demanding evidence of the existence of the HCS with a distribution function having the scaling property given in Eq. (16) is provided by the results to be reported in this paper.

The temperature dependence of the cooling rate for the $\mathrm{HCS}, \zeta_{h}(t)$, can be determined by dimensional analysis to be $\zeta_{h}\left[n_{h}, T_{h}(t)\right] \propto T_{h}(t)^{1 / 2}$. Now, Eq. (14), particularized for the 
HCS of a system of hard spheres or disks, can be integrated, to obtain the time dependence of the temperature

$$
T_{h}(t)=T_{h}\left(t^{\prime}\right)\left[1+\frac{\zeta^{*} v_{0}\left(t^{\prime}\right)\left(t-t^{\prime}\right)}{2 \ell}\right]^{-2},
$$

with

$$
\zeta^{*} \equiv \frac{\ell \zeta_{h}(t)}{v_{0}(t)}
$$

being a dimensionless time-independent cooling rate. This algebraic decay of the temperature of the HCS is known as the Haff law [21].

For the analysis of the HCS, it is useful to introduce the dimensionless time scale $s$ defined through

$$
s(t)=\int_{0}^{t} d t^{\prime} \frac{v_{0}\left(t^{\prime}\right)}{\ell} .
$$

Therefore, $s$ is proportional to the accumulated average number of collisions per particle in the time interval $(0, t)$. In terms of this new time variable, the cooling law (17) becomes

$$
T(s)=T\left(s^{\prime}\right) e^{-\left(s-s^{\prime}\right) \zeta^{*}} .
$$

The $t$ and $s$ time scales are related through

$$
s=\frac{2}{\zeta^{*}} \ln \left[1+\frac{\zeta_{h}(0)}{2} t\right],
$$

as can be directly seen by comparison of Eqs. (17) and (20) or, equivalently, by direct integration of Eq. (19).

\section{TIME-CORRELATION FUNCTIONS IN THE HCS}

As indicated in the previous section, the scaling property of the distribution function of the HCS implies that the time-dependence of many macroscopic properties of the system can be identified without carrying out explicit calculations. Let $A(\Gamma)$ be a homogeneous function of degree $a$ of the velocities of the particles. Then, it is

$$
\begin{aligned}
A(\Gamma) \equiv A\left(\left\{\boldsymbol{q}_{r}, \boldsymbol{v}_{r} ; r=1, \ldots, N\right\}\right) & \equiv A\left(\left\{\ell \boldsymbol{q}_{r}^{*}, v_{0}(t) \boldsymbol{v}_{r}^{*} ; r=1, \ldots, N\right\}\right) \\
& =v_{0}^{a}(t) A\left(\left\{\ell \boldsymbol{q}_{r}^{*}, \boldsymbol{v}_{r}^{*} ; r=1, \ldots, N\right\}\right),
\end{aligned}
$$

where $\boldsymbol{q}_{r}^{*} \equiv \boldsymbol{q}_{r} / \ell$ and $\boldsymbol{v}_{r}^{*} \equiv \boldsymbol{v}_{r} / v_{0}(t)$. Examples of this kind of properties are the center of mass velocity or the total energy of the system. The average value of $A$ in the HCS is

$$
\langle A(t)\rangle_{h}=\int d \Gamma A(\Gamma) \rho_{h}(\Gamma, t)=v_{0}^{a}(t)\langle A\rangle_{h}^{*},
$$


where

$$
\langle A\rangle_{h}^{*} \equiv \int d \Gamma^{*} \rho_{h}^{*}\left(\Gamma^{*}\right) A\left(\left\{\ell \boldsymbol{q}_{r}^{*}, \boldsymbol{v}_{r}^{*} ; r=1, \ldots, N\right\}\right),
$$

and $\Gamma^{*} \equiv\left\{\boldsymbol{q}_{r}^{*}, \boldsymbol{v}_{r}^{*} ; r=1, \ldots, N\right\}$. Thus all the time dependence of $\langle A(t)\rangle_{h}$ is in the factor $v_{0}^{a}(t)$.

Suppose next that $B(\Gamma)$ is also a homogeneous function of the velocities of degree $b$,

$$
B(\Gamma)=v_{0}^{b}(t) B\left(\left\{\ell \boldsymbol{q}_{r}^{*}, \boldsymbol{v}_{r}^{*} ; r=1, \ldots, N\right\}\right),
$$

and consider the HCS time-correlation function for $A$ and $B$ defined as

$$
C_{A B}\left(t, t^{\prime}\right) \equiv\left\langle A(t) B\left(t^{\prime}\right)\right\rangle_{h}-\langle A(t)\rangle_{h}\left\langle B\left(t^{\prime}\right)\right\rangle_{h},
$$

for $t \geq t^{\prime} \geq 0$. By carrying out the transformation to dimensionless variables, it can be shown that [14]

$$
\left\langle A(t) B\left(t^{\prime}\right)\right\rangle_{h}=v_{0}^{a}(t) v_{0}^{b}\left(t^{\prime}\right)\left\langle A\left(s-s^{\prime}\right) B\right\rangle_{h}^{*},
$$

with

$$
\langle A(s) B\rangle_{h}^{*}=\int d \Gamma^{*} \rho_{h}^{*}\left(\Gamma^{*}\right) A\left(\left\{\ell \boldsymbol{q}_{r}^{*}, \boldsymbol{v}_{r}^{*}\right\}, s\right) B\left(\left\{\ell \boldsymbol{q}_{r}^{*}, \boldsymbol{v}_{r}^{*}\right\}\right) .
$$

Here

$$
A\left(\left\{\ell \boldsymbol{q}_{r}^{*}, \boldsymbol{v}_{r}^{*}\right\}, s\right)=e^{s \mathcal{L}^{*}} A\left(\left\{\ell \boldsymbol{q}_{r}^{*}, \boldsymbol{v}_{r}^{*}\right\}\right),
$$

where $\mathcal{L}^{*}$ is the new generator for the dynamics of the phase functions,

$$
\begin{gathered}
\mathcal{L}^{*}\left(\Gamma^{*}\right) \equiv \frac{\zeta^{*}}{2} \sum_{r=1}^{N} \boldsymbol{v}_{r}^{*} \cdot \frac{\partial}{\partial \boldsymbol{v}_{r}^{*}}+L^{*}\left(\Gamma^{*}\right), \\
L^{*}\left(\Gamma^{*}\right)=\frac{\ell}{v_{0}(t)} L(\Gamma)=[L(\Gamma)]_{\left\{\boldsymbol{q}_{r}=\boldsymbol{q}_{r}^{*}, \boldsymbol{v}_{r}=\boldsymbol{v}_{r}^{*}\right\}, \sigma=\sigma^{*}} .
\end{gathered}
$$

The first term on the right hand side of Eq. (30), is due to the time-dependent scaling of the velocities with $v_{0}(t)$. Use of Eqs. (23) and (27) into Eq. (26) yields

$$
\begin{gathered}
C_{A B}\left(t, t^{\prime}\right)=v_{0}^{a}(t) v_{0}^{b}\left(t^{\prime}\right) C_{A B}^{*}\left(s-s^{\prime}\right), \\
C_{A B}^{*}(s)=\langle A(s) B\rangle_{h}^{*}-\langle A\rangle_{h}^{*}\langle B\rangle_{h}^{*} .
\end{gathered}
$$

To identify the aging phenomena clearer, it is convenient to normalize the correlation function to unity for $t=t^{\prime}$, by defining a relaxation function $\phi_{A B}\left(t, t^{\prime}\right)$ as

$$
\begin{aligned}
\phi_{A B}\left(t, t^{\prime}\right) & \equiv \frac{C_{A B}\left(t, t^{\prime}\right)}{C_{A B}\left(t^{\prime}, t^{\prime}\right)}=\left[\frac{v_{0}(t)}{v_{0}\left(t^{\prime}\right)}\right]^{a} \phi_{A B}^{*}\left(s-s^{\prime} ; \zeta^{*}\right) \\
& =e^{-\left(s-s^{\prime}\right) \frac{a \zeta^{*}}{2}} \phi_{A B}^{*}\left(s-s^{\prime} ; \zeta^{*}\right) .
\end{aligned}
$$


Upon writing the last equality above, use has been made of Eq. (20). The dimensionless relaxation function $\phi_{A B}^{*}$ above is

$$
\phi_{A B}^{*}\left(s ; \zeta^{*}\right)=\frac{\langle A(s) B\rangle_{h}^{*}-\langle A\rangle_{h}^{*}\langle B\rangle_{h}^{*}}{\langle A B\rangle_{h}^{*}-\langle A\rangle_{h}^{*}\langle B\rangle_{h}^{*}} .
$$

It follows from Eq. (34) that the two-time correlation function depends on time only through the difference $s-s^{\prime}$. Consequently, there is no aging when time is measured in the dimensionless scale $s$. In ref. [16], the aging property of the velocity time-autocorrelation function of a granular gas of inelastic hard particles was investigated by means of MD simulations. Time was measured by the average cumulated number of collisions per particles that, as said above, is proportional to the dimensionless time scale $s$ used here. The simulations indicate that the velocity autocorrelation function, $C_{v v}\left(s, s^{\prime}\right)$ in the language used here, depends both on $s^{\prime}$ and $s-s^{\prime}$, and from this feature the authors conclude that the system exhibits aging. Of course, the analysis developed here applies for the case of the velocity time-autocorrelation function, corresponding to $a=b=1$ and, therefore, aging should not be expected according to the results derived above. This apparent discrepancy seems to occur because of a wrong use of the physical concept of aging in ref. [16]. As pointed out in the Introduction, for the existence of aging, it is not enough the dependence of the time-correlation function on both $s^{\prime}$ and $s-s^{\prime}$. In fact, the analysis developed here leads to

$$
C_{A B}\left(s, s^{\prime}\right)=C_{A B}\left(s^{\prime}, s^{\prime}\right) \phi_{A B}\left(s, s^{\prime}\right)=f\left(s^{\prime}\right) e^{-\frac{a \zeta^{*}}{2}\left(s-s^{\prime}\right)} \phi_{A B}^{*}\left(s-s^{\prime} ; \zeta^{*}\right) .
$$

Nevertheless, all the dependence on $s^{\prime}$ occurs in the prefactor $f\left(s^{\prime}\right)=C_{A B}\left(s^{\prime}, s^{\prime}\right)$ and, therefore, there is no real aging, since the decaying rate is always the same and only the initial value changes with $s^{\prime}$ for constant $s-s^{\prime}$.

On the other hand, the aging phenomenon shows up in the original time scale $t$ in the limits $\zeta_{h}(0) t^{\prime} \gg 1$ and $\zeta_{h}(0) t \gg 1$. In this regime, it follows from Eq. (21) that

$$
s-s^{\prime} \sim \frac{2}{\zeta^{*}} \ln \frac{t}{t^{6}}
$$

and, therefore, Eq. (34) takes the form

$$
\phi_{A B}\left(t, t^{\prime}\right)=\left(\frac{t^{\prime}}{t}\right)^{a} \phi_{A B}^{*}\left[\frac{2}{\zeta^{*}} \ln \frac{t^{\prime}}{t} ; \zeta^{*}\right] \equiv F\left(\frac{t}{t^{\prime}} ; \zeta^{*}\right),
$$

valid for $t, t^{\prime} \gg \zeta_{h}(0)^{-1}$. This result is the mathematical expression of the aging phenomenon. The normalized time-correlation function depends on the initial and final times, $t^{\prime}$ and $t$, only 
through their quotient $t / t^{\prime}$, so the system exhibits full aging, as defined in the Introduction. The remaining parameter determining the long time behavior of the correlation function is the dimensionless cooling rate $\zeta^{*}$. It is worth to stress the generality of this result. No limitation on the degree of inelasticity or density has been introduced. In fact, both magnitudes are relevant in determining, through the value of $\zeta(0)$, the time region in which the aging behavior predicted by Eq. (36) is to be expected. The only hypothesis made here is the existence of the HCS with a distribution function having the scaling form given in Eq. (16). Of course, the system is assumed to stay in that state for all the relaxation time $t$ considered. This requires that the HCS, in addition to exist, be stable, at least in a determined region of parameters. The extensive measurements of the velocity correlation function by means of MD simulations reported in ref. [22] show that it is possible to cover a wide range of density and inelasticity in which the observed homogenous state appears to be stable. This is confirmed by the simulation results to be presented here in the next section.

\section{ENERGY TIME CORRELATION FUNCTION IN THE HCS FOR A DILUTE GRANULAR GAS}

Theoretical predictions for the explicit form of the function $\phi_{A B}^{*}\left(s, \zeta^{*}\right)$ are scarce in the literature. An exception is the total energy autocorrelation function, $C_{E E}\left(t, t^{\prime}\right)$, for a dilute granular gas of hard particles. By projecting the Liouville equation onto the hydrodynamic modes in the low density limit, an analytical expression for $C_{E E}$ was derived in ref. [15],

$$
C_{E E}\left(t, t^{\prime}\right)=N T(t) T\left(t^{\prime}\right) e(\alpha) e^{-\left(s-s^{\prime}\right) \frac{\zeta^{*}}{2}}
$$

where $e(\alpha)$ is a given function of only the coefficient of restitution. Then,

$$
\phi_{E E}\left(t, t^{\prime}\right) \equiv \frac{C_{E E}\left(t, t^{\prime}\right)}{C_{E E}\left(t^{\prime}, t^{\prime}\right)}=\frac{T(t)}{T\left(t^{\prime}\right)} e^{-\left(s-s^{\prime}\right) \frac{s^{*}}{2}} .
$$

Since the energy $E$ is a homogenous function of degree $a=2$ of the velocities of the particles,

comparison of the above expression with the general result given in Eq. (34) leads to the identification

$$
\phi_{E E}^{*}\left(s ; \zeta^{*}\right)=e^{-\frac{\zeta^{*}}{2} s} .
$$


Therefore, using Eq. (38), it follows that the aging behavior of the total energy of a dilute granular gas of hard particles is given by

$$
\phi_{E E}\left(t, t^{\prime}\right)=e^{-\frac{3 \zeta^{*}}{2}\left(s-s^{\prime}\right)} \sim\left(\frac{t^{\prime}}{t}\right)^{3},
$$

for $t, t^{\prime} \gg \zeta_{h}(0)^{-1}$. An explicit expression for the cooling rate of a dilute granular gas has been derived from the Boltzmann equation in the so-called first Sonine approximation [23]. This expression has been shown to accurately agree with the numerical results obtained by means of the direct simulation method of the Boltzmann equation [20, 24] in the thermal velocity region, i.e. for velocities of the order of $v_{0}(t)$.

Due to the simplicity of $\phi_{E E}^{*}$ in the present case, the asymptotic form of the normalized time correlation function for the energy, $\phi_{E E}\left(t, t^{\prime}\right)$, does not depend on the value of $\zeta^{*}$, what implies that it is independent of the density $n_{h}$ and of the coefficient of restitution $\alpha$. Consequently, if $\phi_{E E}\left(t, t^{\prime}\right)$ is plotted as a function of $t / t^{\prime}$ for times in the range $t, t^{\prime} \gg \zeta(0)^{-1}$, the curves corresponding to different densities and inelasticities should tend to collapse on a unique one. Of course, the density range to which this result applies is restricted by the validity of the Boltzmann description.

To check the above theoretical predictions, we have performed MD simulations of a system of inelastic hard disks $(d=2)$. In Fig. 1, results obtained for a system of $N=10^{3}$ particles with coefficient of normal restitution $\alpha=0.95$ and density $n_{h} \sigma^{2}=0.02$ are reported. For these values of the parameters, the HCS is stable, since the critical length is larger than the size of the system and, therefore, the velocity vortices and high density clusters characteristic of the clustering instability [25] cannot develop. Of course, in all the simulations it has been verified that the system remains homogeneous. The reported results have been averaged over 1200 trajectories of the system. The several curves correspond to different values of $\zeta_{h}(0) t^{\prime}$, as indicated in the figure. The value of $\zeta_{h}(0)$ has been estimated by using the low density expressions derived in [23].

In agreement with the analysis presented here, it is observed that as $t^{\prime}$ increases the timecorrelation function approaches a form that depends only on the value of the time ratio $t / t^{\prime}$. More precisely, for $\zeta_{h}(0) t^{\prime} \gtrsim 24$, all the plotted curves coincide within the statistical uncertainties. Note that the latter increase as the value of $t$ grows, for a given value of $t^{\prime}$. Moreover, the asymptotic curve agrees with the theoretical prediction given by Eq. (42), whose graphical representation is the solid line in the figure. This good accuracy is consistent 




FIG. 1: Dimensionless time self-correlation function of the total energy, $\phi_{E E}\left(t, t^{\prime}\right)$, for a dilute granular gas of inelastic hard disks in the HCS. The density is $n_{h} \sigma^{2}=0.02$ and the restitution coefficient $\alpha=0.95$. The different symbols correspond to different values of the initial time, as indicated. The solid line is the theoretical prediction describing the full aging phenomenon, Eq. (42).

with the value of the density of the system, that is small enough as to expect a low density description, at the level of the Boltzmann equation, to apply.

In Fig. 2, a similar plot is given, but now two systems, one with $n_{h} \sigma^{2}=0.02, \alpha=0.85$, and the other with $n_{h} \sigma^{2}=0.1, \alpha=0.98$, are considered. For the sake of clearness, only simulation data corresponding to large waiting times have been included. Again, a good agreement with the behavior predicted by Eq. (42) is observed. The same behavior has been obtained for other densities between the two above values. This confirms the independence of $\phi_{E E}\left(t, t^{\prime}\right)$ from the density and the inelasticity at low density. In fact, the good agreement observed for $n_{h} \sigma^{2}=0.1$ must be stressed, because at this density the gas can not be considered as very dilute.

Prompted by the results for $n_{h} \sigma^{2}=0.1$ given in Fig. 2, the correlation function $\phi_{E E}\left(t, t^{\prime}\right)$ has also been evaluated at a definitely non small density, namely $n_{h} \sigma^{2}=0.2$. For this value, density corrections to the low density behavior are clearly identified in most of the 


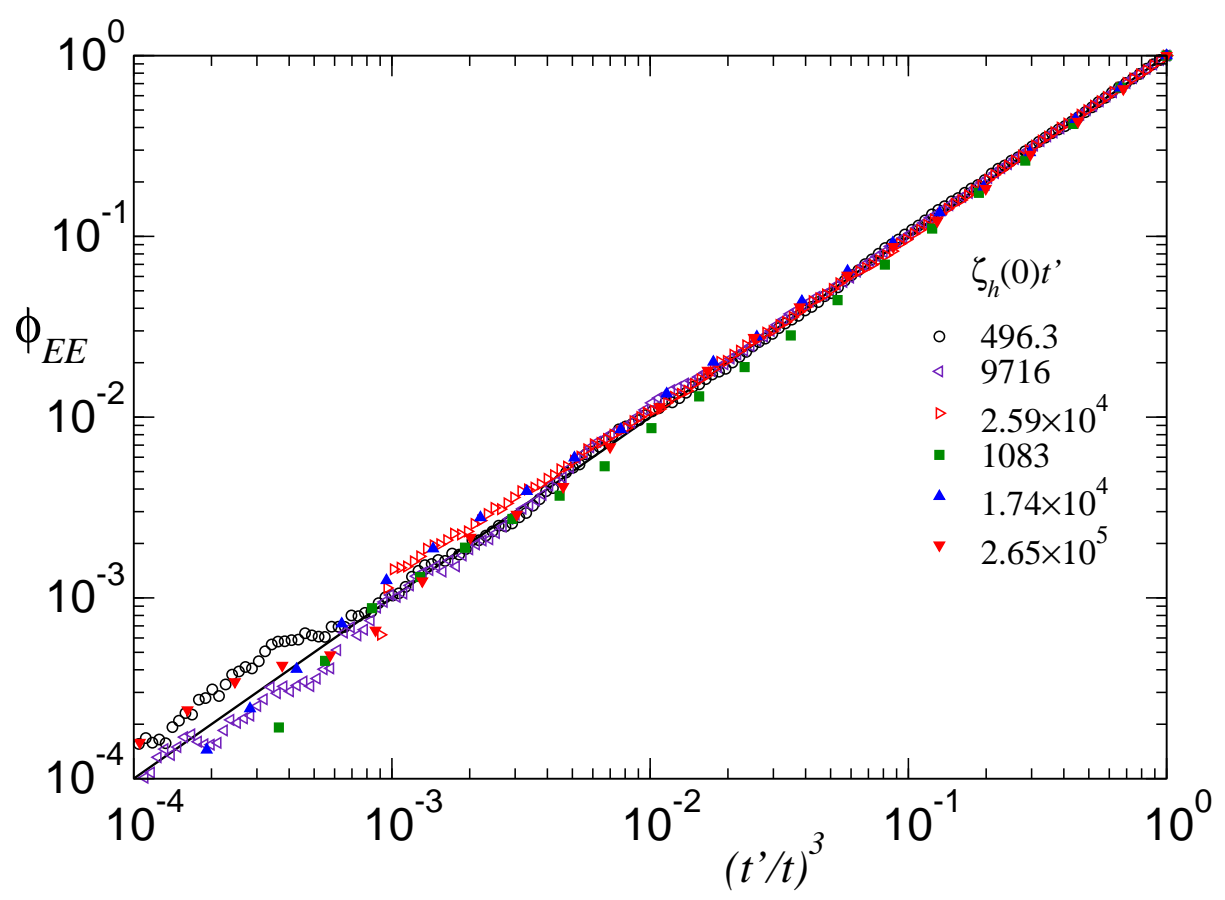

FIG. 2: The same as in Fig. 1, but for different values of the (low) density and of the restitution coefficient. Filled symbols correspond to a system with $n_{h} \sigma^{2}=0.02$ and $\alpha=0.85$, while empty symbols refer to a system with $n_{h} \sigma^{2}=0.1$ and $\alpha=0.98$.

equilibrium properties of a molecular gas. It must be mentioned that, in order to keep the system well inside the stable region of parameters and with a number large enough of particles, the value of $\alpha$ must be rather close to unity. Moreover, they have been averaged over 1500 trajectories. The results shown in Fig. 3 have been obtained with $\alpha=0.98$ and $N=700$. Once again, a tendency towards a behavior depending only on the ratio $t / t^{\prime}$ as $t^{\prime}$ increases is clearly identified, i.e. the system exhibits full aging. Besides, and rather surprisingly, the aging phenomenon seems to be accurately described over several decades by the law $\left(t^{\prime} / t\right)^{3}$, that was obtained here in the context of very dilute granular gases (Boltzmann limit).

\section{DISCUSSION}

The objective here has been to explore the existence of aging in the homogeneous cooling state of a granular gas. This has been done by exploiting the assumed scaling property of the $N$-particle distribution of this state for a system of inelastic hard spheres or disks. In 


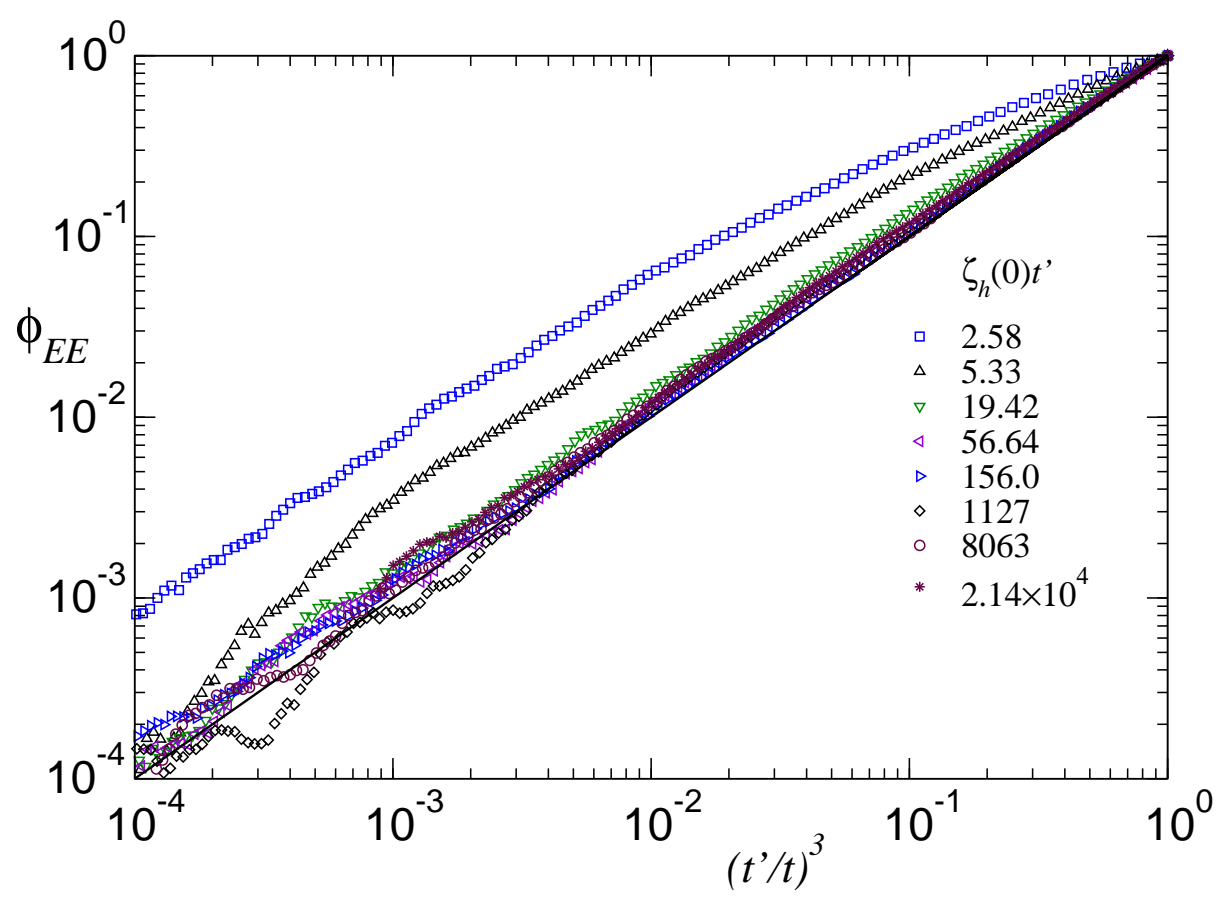

FIG. 3: The same as in Fig. 1, but for $n_{h} \sigma^{2}=0.2$ and $\alpha=0.98$. A clear tendency towards a function depending only on $t / t^{\prime}$, characteristic of full aging, is observed as $t^{\prime}$ increases.

fact, the presence of aging and its specific form turn out to be directly associated to the scaling property of the distribution [14, 15]. From this perspective, the agreement between molecular dynamics simulations and the theory discussed in this paper, is an almost direct proof of the existence of the homogeneous cooling state also at the level of the full many body pseudo-Liouville equation. This would extend the rather well established fact that the inelastic Boltzmann and Enskog equations have such a solution, consistently with some previous results [19, 20].

Granular media are inherently non-equilibrium systems due to the lack of energy conservation in the interactions between grains. They present a very rich phenomenology which, sometimes, is similar to that of normal, molecular systems. Moreover, it has been verified in the last years that the methods of kinetic theory and non-equilibrium statistical mechanics developed for normal fluids, can be extended to granular fluids, yielding to results having an analogous structure. There are also significant differences, but they are well understood as consequences of the inelasticity. One characteristic feature of granular systems is that quite often they exhibit the phenomena in a much simpler context than molecular systems. This refers to both, theoretical and experimental views. In this paper, the simplicity of the HCS 
of a granular fluid has allowed the identification of aging and the derivation of its explicit form in the dilute limit, Eq. (42), for the correlation of the total energy of the system. The analytical expression, that corresponds to the so-called full aging, has been shown to be in perfect agreement with MD simulation results. In particular, the long time limit of the normalized time-correlation function of the total energy is independent of the inelasticity and the density. Quite interestingly, the simulations indicate that this independence seems to extend to densities beyond the dilute limit.

To really appreciate the results presented here, it is important to differentiate between both the existence of aging and the specific, particular, form of the law governing it. Equation (38) implies the existence of full aging in the system, i.e. the normalized time-correlation function of the properties $A$ and $B$ is not a function of the time difference $t-t^{\prime}$ and depends on the time ratio $t / t^{\prime}$. The only necessary condition to derive this equation is the existence of the HCS itself, as discussed above. On the other hand, identification of the function $F$ in Eq. (38) requires more detailed additional analysis, which has been carried out only in the low density limit up to now. The above leads to Eq. (42) in the particular case of the properties $A$ and $B$ being both the total (kinetic) energy of the system. In this sense, the results presented in Fig. 3 strongly support the existence of the HCS and the scaling property of its $N$-particle distribution function at high densities, independently of whether or not the convergence occurs towards the power law given by Eq. (42), as suggested by the simulation results.

In real granular gases, the restitution coefficient is not constant, but it depends on the impact relative velocity. Then, the distribution function of the HCS does not scale in the form given by Eq. (16) and, therefore, the discussion in the present paper does not apply in principle, although it can provide an accurate approximation to the actual behavior of the system. To be more precise, consider a given model of granular gas with velocity-dependent coefficient of normal restitution. Now, the problem being addressed has two energy scales. One is the total energy per particle or, equivalently, the cooling temperature $T_{h}(t)$. The other energy scale, $\epsilon$, is fixed by some property of the specific collision model. Define a dimensionless parameter

$$
\epsilon^{*} \equiv \frac{\epsilon}{m v_{0}^{2}\left(T_{h}\right)} .
$$

For hard spheres, $\epsilon=0$ and so $\epsilon^{*}=0$. It is in this limit when the distribution function of the HCS has the scaling property (16) [26]. For $\epsilon^{*}>0$, the scaling does not hold exactly, 
but it can be an appropriate description as long as $\epsilon^{*} \ll 1$, i.e. the interaction be sufficiently hard and/or the kinetic energy of the particles be sufficiently large.

\section{ACKNOWLEDGEMENTS}

This research was partially supported by the Ministerio de Educación y Ciencia (Spain) through Grant No. BFM2005-01398.

[1] J.-P. Bouchaud, L.F. Cugliandolo, J. Kurchan, and M. Mézard, in Spin Glasses and Random Fields, edited by A.P. Young (Word Scientific , Singapore, 1997), p. 161.

[2] J.P. Bouchaud, J. Phys. I 2, 1705 (1992).

[3] L.F. Cugliandolo, J. Kurchan, and F. Ritort, Phys. Rev. B 49, 6331 (1994).

[4] A. Prados and J.J. Brey, Europhys. Lett. 40, 13 (1997).

[5] C. Godrèche and J.M. Luck, J. Phys. A: Math. Gen. 33, 1151 (2000).

[6] D.S. Fisher and D.A. Huse, Phys. Rev. B 38, 373 (1988).

[7] A.J. Bray, Adv. Phys. 43, 357 (1994).

[8] J.B. Knight, C.G. Fandrich, C.N. Lau, H.M. Jaeger, and S.R. Nagel, Phys. Rev. E 51, 3957 (1995).

[9] E.R. Novak, J.B. Knight, E. Ben-Naim, H.M. Jager, and S.R. Nagel, Phys. Rev. E 57, 1971 (1998).

[10] G.W. Scherer, Relaxation in Glass and Composites (Wiley, New York, 1986).

[11] C. Josserand, A. Tkackenko, D.M. Mueth, and H.M. Jaeger, Phys. Rev. Lett. 85, 3632 (2000).

[12] M. Nicodemi and A. Coniglio, Phys. Rev. Lett. 82, 916 (1999).

[13] J.J. Brey and A. Prados, Phys. Rev. E 63, 061301 (2001).

[14] J.W. Dufty, J.J. Brey, and J. Lutsko, Phys. Rev. E 65, 051303 (2002).

[15] J.J. Brey, M.I. García de Soria, P. Maynar, and M.J. Ruiz-Montero, Phys. Rev. E 70, 011302 (2004).

[16] S.R. Ahmad and S. Puri, Phys. Rev. E 75, 031302 (2007).

[17] J.J. Brey, J.W. Dufty, and A. Santos, J. Stat. Phys. 87, 1051 (1997).

[18] I. Goldhirsch and T.P.C. van Noije, Phys. Rev. E 61, 3241 (2000). 
[19] J.F. Lutsko, Phys. Rev. E 63, 061211 (2001).

[20] J.J. Brey, M.J. Ruiz-Montero, and F. Moreno, Phys. Rev. E 69, 051303 (2004).

[21] P.K. Haff, J. Fluid Mech. 134, 401 (1983).

[22] J. Lutsko, J.J. Brey, and J.W. Dufty, Phys. Rev. E 65, 051304 (2002).

[23] A. Goldsthein and M. Shapiro, J. Fluid Mech. 282, 75 (1995); T.P.C. van Noije and M.H. Ernst, Granular Matter 1, 57 (1998).

[24] J.J. Brey, M.J. Ruiz-Montero, and D. Cubero, Phys. Rev. E 54, 3664 (1996).

[25] I. Goldhirsch and G. Zanetti, Phys. Rev. Lett. 70, 1619 (1993).

[26] J.W. Dufty, A. Baskaran, and J.J. Brey arXiv:cond-mat/0612408. 